\title{
An experimental study of ascorbic acid effects in acute renal failure under general anesthesia ${ }^{1}$
}

Omur Ozturk', Sefer Ustebay", Huseyin Avni Eroglu'I", Murat Günay'v, Yasemen Adaliv, illksen Donmez ${ }^{\mathrm{VI}}$, Mesut Erbas ${ }^{\mathrm{VII}}$

'Assistant Professor, Department of Anesthesiology and Reanimation, Faculty of Medicine, Canakkale Onsekiz Mart University, Turkey. Conception, design, scientific and intellectual content of the study; acquisition of data; statistical analysis; manuscript writing; final approval.

"Assistant Professor, Department of Pediatrics, Faculty of Medicine, Kafkas University, Kars, Turkey. Analysis of data, technical procedures, manuscript writing.

"'Assistant Professor, Department of Physiology, Faculty of Medicine, Kafkas University, Kars, Turkey. Analysis of data, statistical analysis, technical procedures, critical revision.

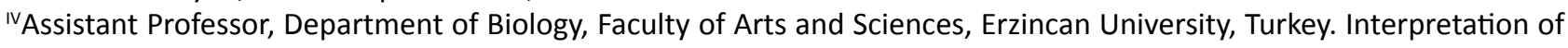
data, biochemical parameters.

${ }^{v}$ Assistant Professor, Department of Pathology, Faculty of Medicine, Kafkas University, Kars, Turkey. Interpretation of data, pathological examinations.

V'Assistant Professor, Department of Anesthesiology and Reanimation, Faculty of Medicine, Kafkas University, Kars, Turkey. Analysis of data, manuscript writing.

VIIAssociate Professor, Department of Anesthesiology and Reanimation, Faculty of Medicine, Canakkale Onsekiz Mart University, Turkey. Manuscript writing, critical revision.

\section{Abstract}

Purpose: To evaluate the preventive effect of ascorbic acid on sevoflurane-induced acute renal failure in an experimental rat model.

Methods: Twenty-four adult male Wistar rats were randomly distributed into three groups. Subjects were allocated into 3 groups: Group I received sevoflurane only, whereas Groups II and III had moderate $(150 \mathrm{mg} / \mathrm{kg})$ and high $(300 \mathrm{mg} / \mathrm{kg})$ doses of AA in addition to sevoflurane, respectively. Rhabdomyolysis and myohemoglobinuric ARF was formed by intramuscular administration of glycerol on the upper hind limb on the $15^{\text {th }}$ minute of inhalation anesthesia. Biochemical parameters consisted of serum levels of blood urea nitrogen, creatinine, neutrophil gelatinase-associated lipocalin (NGAL), total antioxidant capacity (TAC), and protein carbonyl content. Histopathological variables were tubular necrosis, fibrin, and cast formation.

Results: NGAL levels were significantly lower in Group III than Group II and Group I. On the other hand, TAC, PCO, urea and creatinine levels were notably higher in Group I compared with Groups II and III. There was a significant difference between 3 groups on frequencies of acute tubular necrosis $(p=0.003)$, fibrin $(p<0.001)$ and cast $(p<0.001)$. Acute tubular necrosis and fibrin formation were more prominent in Group I. Casts were more common in Groups II and III.

Conclusions: The ascorbic acid serve as a prophylactic agent against renal damage in patients receiving sevoflurane anesthesia and higher doses were associated with more apparent protective effects.

Key words: Ascorbic Acid. Glycerol. Acute Kidney Injury. Anesthesia, General. Rats. 


\section{Introduction}

Acute renal failure (ARF) may occur in the early postoperative period, and its incidence has been reported as $0.1 \%-30 \%$ after various surgical procedures ${ }^{1,2}$. Postoperative ARF has been linked with many factors including perioperative hypotension and nephrotoxin-induced acute tubular necrosis. Type of anesthesia may significantly affect the likelihood of acute renal tubular damage ${ }^{1}$. Volatile anesthetics such as sevoflurane are commonly used in clinical practice, and they have different degrees and sites of metabolism $^{3}$. Metabolic reactions during elimination of volatile anaesthetics may lead to elevation of nephrotoxic inorganic fluoride levels ${ }^{4}$. Notably, even though high doses of inorganic fluoride may lead to proximal tubular necrosis, it may have cytoprotective effect in low doses ${ }^{5}$. Intramuscular glycerol injection causes a nephrotoxic (myohemoglobinuric)/ ATP depletion leading to acute tubular necrosis ${ }^{6}$.

Sevoflurane may affect inflammatory processes and ischemia/reperfusion injury in various organs including kidney ${ }^{7,8}$. It has been reported that sevoflurane may not impair renal function in healthy patients or even in patients with impaired renal functions ${ }^{9}$.

Ascorbic acid (AA) may alleviate the injury due to oxidative stress in kidney, and it may also have a preventive effect against nephrotoxicity ${ }^{10}$. Ascorbic acid has protective and anti-oxidant effects against nephrotoxicity occurring after traumatic muscle injury elicited by glycerol $^{11}$. In recent literature, glycerol administration was found to cause marked renal oxidative stress and severe renal functional and morphological deterioration ${ }^{12}$. The treatment of animals with AA partially corrected the renal dysfunction and morphological impairment. Therefore, they suggested that AA could be a promising candidate for the prevention of rhabdomyolysis-induced ARF. Higher dosages of vitamin C (>20 mg/kg) was supposed to be more beneficial regarding functional and morphological aspects in this model of $\mathrm{ARF}^{12}$.

Neutrophil gelatinase-associated lipocalin (NGAL) has been used as an early predictive biomarker of acute renal injury ${ }^{13}$. Serum levels of urea and creatinine were investigated to assess the nephrotoxicity. Protein carbonyl content (PCO) is an important parameter for evaluation of oxidative damage to proteins. Protein carbonyl content represents an irreversible protein modification, often leading to the inactivation of the proteins and it is a sensitive and an early marker of oxidative stress to tissues ${ }^{14}$.

The aim of the current experimental study was to investigate whether AA may have a preventive or ameliorative effect on acute renal failure in patients who are planned for sevoflurane anesthesia. In this perspective, we comparatively evaluated the histopathological and biochemical outcomes of sevoflurane anesthesia on kidneys with and without $A A$ in an acute tubular necrosis model triggered with glycerol. Exploration of preventive effects of $A A$ may yield new insights to decrease mortality and morbidity in acute renal failure patients who will undergo sevoflurane anesthesia.

\section{- Methods}

Twenty-four adult male Wistar rats (aged 8 to 10 weeks, weighing 200 to 250 g) were included after the approval of our research protocol by the local institutional laboratory review board (date/no). Adherence to the principles declared in the Guide for the Care, and Use of Laboratory Animals (National Institutes of Health, 1985) was provided.

Animals were fed on a standard rat chow diet. Access to water and food was allowed ad libitum. Cages were kept at a temperature of $24 \pm 2{ }^{\circ} \mathrm{C}$ and humidity of $55 \pm 5 \%$ at a 12 -hour light $\&$ dark cycle. Before the study, rats were dehydrated by keeping away from water for 24 
hours. Animals were randomly allocated into 3 experimental groups ( $n=8$ for each group). Group I received sevoflurane only, whereas Groups II and III had moderate (150 mg/ $\mathrm{kg}$ ) and high $(300 \mathrm{mg} / \mathrm{kg}$ ) doses of $A A$ in addition to sevoflurane, respectively. Sedation was made using thiopental $(30 \mathrm{mg} / \mathrm{kg}$ ) intraperitoneally. To measure the invasive blood pressure, the right femoral artery cannulation was performed and, if necessary, the left femoral venous cannulation was performed for fluid infusion. Initially, blood samples $(1 \mathrm{~mL})$ were obtained from the tail veins to determine baseline values of biochemical markers under investigation. Inhalational anesthetics were administered during 4 hours in specially designed conical shaped appropriate mask. For all groups, sevoflurane was introduced at a rate of $2 \mathrm{~L} / \mathrm{min}$ in $100 \%$ oxygen. The flow of oxygen was set at a rate of $4 \mathrm{~L} / \mathrm{min}$ with the anesthesia apparatus. The concentration of sevoflurane was adjusted on the respiratory pattern and heart rate. The same sevoflurane vaporizer was utilized for all rats. Rhabdomyolysis and myohemoglobinuric ARF was formed using modified glycerol model on the 15th minute of inhalation aaesthesia. Glycerol (50\% solution, $9 \mathrm{~mL} / \mathrm{kg}$ ) was intramuscularly applied on the upper hind limb. Ascorbic acid was administered intraperitoneally, and the total volume of injectate was completed to $2 \mathrm{~mL}$ using isotonic saline. After cessation of anesthesia, rats were transferred to a warm, moist and well-aerated medium. Body temperature maintained between $36^{\circ} \mathrm{C}$ to $37^{\circ} \mathrm{C}$ was monitored with a rectal probe. During the experiment, the mean arterial blood pressure was maintained at $90 \pm 5 \mathrm{mmHg}$. At the end of the anesthesia procedure, rats were put in standard cages that allowed rapid recovery from anesthesia and free access to water and food were provided. Twenty-four hours after cessation of anesthesia, rats were sedated with thiopental $(75 \mathrm{mg} / \mathrm{kg}$ ) administered intraperitoneally. Abdomens were opened with a midline incision laparotomy and rats were sacrificed by aortic puncture followed by kidney resection. Blood samples were obtained from abdominal aorta during aortic puncture. Blood samples were centrifuged at $1500 \mathrm{~g}$ for 10 minutes to extract the serum and maintained at $-70^{\circ} \mathrm{C}$ until analysis for levels of creatinine, urea, neutrophil gelatinaseassociated lipocalin (NGAL), total antioxidant capacity (TAC) and protein carbonyl content (PCO).

Both kidneys were dissected and fixed in $10 \%$ neutral formalin solution. After embedding in paraffin, sections of $4 \mu \mathrm{m}$ thickness were prepared. After hematoxylineosin staining, histopathological examination was carried out by a pathologist blinded to data for experimental groups under light microscopy (Olympus BX50; Olympus Optical Co. Ltd., Tokyo, Japan). Renal tissue samples were evaluated regarding fibrin, cast and necrosis. Histopathologic alterations including fibrin, cast and necrotic material in tubuli were graded on a 3-point semiquantitative scale based on a previously described method as: 0 (no changes); 1 (focal, mild changes); 2 (multifocal, intermediate changes) and 3 (extensive, prominent changes) ${ }^{15}$.

\section{Serum studies}

\section{Urea and creatinine levels}

The concentration of urea in the serum was estimated by the urease-glutamate dehydrogenase enzymatic method ${ }^{16}$. Serum creatinine level was measured with a modified Jaffe method ${ }^{17}$. These measurements were performed using an automatic analyzer (Architect c800; Abbott Diagnostics, Lake Forest, IL, USA).

\section{Total antioxidant capacity (TAC)}

Commercially available kits based on the colorimetric method and an autoanalyzer 
machine (Selectera $\mathrm{XL}$, Holland) were used for measurement of serum TAC ${ }^{18}$.

\section{Protein carbonyl content}

Protein carbonyl content was evaluated spectrophotometrically by the method described by Reznick and Packer ${ }^{19}$. The PCO reacts with 2,4-dinitrophenyl hydrazine (DNPH) and yields chromophoric dinitrophenylhydrazones. $0.5 \mathrm{ml}$ of sample (1-2 mg) was mixed with an equal volume of $10 \mathrm{mM}$ DNPH in $2 \mathrm{~N} \mathrm{HCl}$. Following incubation for 1 hour, it was shaken intermittently at room temperature. A corresponding blank was performed by adding only $2 \mathrm{~N} \mathrm{HCl}$ to the sample. After incubation, the mixture was precipitated with $10 \%$ trichloroacetic acid and centrifuged. The precipitate was washed twice with ethanol: ethyl acetate (1:1) and dissolved in $1 \mathrm{ml}$ of $6 \mathrm{M}$ guanidine $\mathrm{HCl}$, centrifuged at low speed and the supernatant was read at $366 \mathrm{~nm}$. The difference in absorbance between the DNPH treated and $\mathrm{HCl}$-treated sample was determined and expressed as nmoles of carbonyl groups per mg of protein.

Neutrophil gelatinase-associated lipocalin (NGAL) level

Quantitative sandwich ELISA method was used for estimation of NGAL levels. Rat monoclonal antibody (MAB1757, R\&D Systems, USA) against NGAL were used as capturing and detecting antibodies. Recombinant NGAL (RC9209, Randox Laboratories, $U K$ ) was used as a standard (diluted from 1 to $1000 \mu \mathrm{g} / \mathrm{L}$ ) to assess NGAL concentrations in $100 \mu \mathrm{L}$ of serum samples ${ }^{20}$.

\section{Drugs}

Glycerol and AA were purchased from Merck KGaA, and Roche Chemicals and Pharmaceutics, respectively.

\section{Outcome parameters}

The biochemical analysis involved analysis of serum levels of urea, creatinine, PCO, NGAL, and TAC within every group and between groups. Histopathologically, presence, and severity of cast and fibrin formation, as well as necrotic material in renal tubule, were assessed based on a previously described 4-point semiquantitative scale as 0 (no changes); 1 (focal, mild changes); 2 (multifocal, intermediate changes) and 3 (extensive, prominent changes ${ }^{15}$.

\section{Statistical analysis}

Our data was analyzed using IBM Statistical Package for Social Sciences Statistics 20 software (SPSS Inc., Chicago, IL, USA). Histopathological data were expressed as descriptive variables in \%. Wilcoxon test was used to assess the differences within each group before and after the procedure. Comparison of biochemical parameters between groups was carried out using Kruskal-Wallis test. A p value less than 0.05 was considered as statistically significant.

\section{- Results}

As shown in Table 1, NGAL levels were increased significantly in 3 groups after the procedure. Total antioxidant capacity was higher after the procedure in Groups II and III only. Protein carbonyl content, which is an indicator of oxidative stress injury, was decreased in Group III after the intervention. Serum levels of urea and creatinine were increased remarkably in 3 groups after the procedure. 
Table 1 - Levels of neutrophil gelatinase associated lipocalin, total antioxidant capacity, protein carbonyl content, urea and creatinine within each of 3 experimental groups before and after the procedure.

\begin{tabular}{llll} 
Group & Before & After & p-value \\
& Neutrophil gelatinase associated lipocalin $(\mu \mathrm{g} / \mathrm{L})$ & $0.018^{*}$ \\
II & $11.93 \pm 4.91$ & $51.35 \pm 14.71$ & $0.012^{*}$ \\
III & $9.59 \pm 2.55$ & $43.19 \pm 17.07$ & $0.012^{*}$ \\
& $9.09 \pm 3.25$ & $32.15 \pm 6.29$ & \\
I & Total antioxidant capacity $(\mathrm{IU} / \mathrm{ml})$ & & 0.091 \\
II & $1.69 \pm 1.01$ & $2.28 \pm 0.51$ & $0.012^{*}$ \\
III & $1.21 \pm 0.45$ & $2.43 \pm 0.58$ & $0.012^{*}$ \\
& $1.67 \pm 0.54$ & $3.16 \pm 0.52$ & \\
I & Protein carbonyl content $(\mathrm{nmol} / \mathrm{mg}$ protein) & 0.866 \\
II & $85.10 \pm 14.47$ & $87.18 \pm 15.93$ & 0.093 \\
III & $70.78 \pm 11.17$ & $77.29 \pm 5.67$ & $0.036^{*}$ \\
& $71.04 \pm 5.27$ & $64.47 \pm 3.75$ & \\
I & Urea (mmol/L) & & $0.028^{*}$ \\
II & $44.86 \pm 33.15$ & $117.00 \pm 8.04$ & $0.011^{*}$ \\
III & $30.75 \pm 4.27$ & $73.12 \pm 4.36$ & $0.011^{*}$ \\
& $33.87 \pm 1.96$ & $62.25 \pm 4.59$ & \\
I & Creatinine ( $\mu$ mol/L) & & $0.018^{*}$ \\
II & $0.57 \pm 0.07$ & $1.26 \pm 0.18$ & $0.012^{*}$ \\
III & $0.50 \pm 0.05$ & $0.95 \pm 0.08$ & $0.012^{*}$ \\
\hline
\end{tabular}

*Statistically significant

Table 2 presents results of serum NGAL, TAC, PCO, urea and creatinine levels in 3 groups under investigation. Accordingly, NGAL levels were significantly lower in Group III than Group
II and Group I. On the other hand, TAC, urea, and creatinine levels were notably higher in Group I compared with Groups II and III.

Table 2 - Comparison of biochemical parameters before and after the intervention between 3 groups.

\begin{tabular}{lllllllllll}
\hline \multicolumn{3}{c}{ Before } & \multicolumn{8}{c}{ After } \\
& NGAL & TAC & PCO & Urea & Creatinine & NGAL & TAC & PCO & Urea & Creatinine \\
\hline Chi-square & 1.947 & 1.783 & 5.473 & 3.832 & 5.323 & 12.067 & 7.991 & 15.472 & 18.919 & 17.989 \\
Df & 2 & 2 & 2 & 2 & 2 & 2 & 2 & 2 & 2 & 2 \\
Asymp. Sig. & 0.378 & 0.410 & 0.065 & 0.147 & 0.070 & $0.002^{*}$ & $0.018^{*}$ & $<0.001^{*}$ & $<0.001^{*}$ & $<0.001^{*}$ \\
\hline
\end{tabular}

*Statistically significant; df: degree of freedom; NGAL: neutrophil gelatinase associated lipocalin; TAC: total antioxidant capacity; PCO: protein carbonyl content) 
Pearson Chi-square test indicated that there was a significant difference between 3 groups on frequencies of acute tubular necrosis $(p=0.003)$, fibrin $(p<0.001)$ and cast $(p<0.001)$. However, a number of subjects in each cell is small, and only observational interpretations based on descriptive data may provide reliable information. In this perspective, it can be postulated that tubular necrosis and fibrin formation were more prominent in Group I. In contrary, casts were more common in Groups II and III. Group III exhibited a less frequent and lesser degree of histopathological alterations than Group II (Table 3).

Table 3 - Distribution of histopathological alterations including tubular necrosis, fibrin and cast in 3 experimental groups.

\begin{tabular}{llllll} 
Group & \multicolumn{2}{l}{ Tubular necrosis } & & & \\
& None & Mild & Intermediate & Extensive & Total \\
\hline I & 0 & 0 & 4 & 3 & 7 \\
II & 0 & 4 & 4 & 0 & 8 \\
III & 2 & 6 & 0 & 0 & 8 \\
Total & 2 & 10 & 8 & 3 & 23 \\
\hline Group & Fibrin & & & & Total \\
& None & Mild & Intermediate & Extensive & 7 \\
\hline I & 0 & 0 & 0 & 7 & 8 \\
II & 0 & 0 & 7 & 1 & 8 \\
III & 0 & 8 & 0 & 0 & 23 \\
Total & 0 & 8 & 7 & 8 & Total \\
\hline Group & Cast & & & & 7 \\
& None & Mild & Intermediate & Extensive & 8 \\
\hline I & 0 & 0 & 7 & 0 & 8 \\
II & 0 & 2 & 6 & 0 & 23 \\
III & 0 & 2 & 6 & 0 & 0 \\
Total & 0 & 4 & 19 & & \\
\hline
\end{tabular}

\section{- Discussion}

Ascorbic acid is a biologically important reducing substances, and it acts as an antioxidant that provides defense against free radicals ${ }^{21}$. Its protective effect against nephrotoxicity and apoptosis caused by colistin has been demonstrated in experimental trials $^{12}$. Moreover, it has been shown that $A A$ ameliorates myonecrosis and it displays antiaging activities ${ }^{22}$. These beneficial effects of AA have been linked with regulation of the hyperproliferative tissue response including kidneys ${ }^{10}$.
The main objective of this study was to investigate the prophylactic role of AA in ARF patients undergoing sevoflurane anesthesia. Our results demonstrated that both low and high doses of $A A$ might provide protection against nephrotoxicity which may occur in the postoperative period after sevoflurane anesthesia. Though both low and high doses of AA improved total antioxidant capacity, PCO was decreased only the group receiving a high dose of $A A$. This reminds that high-dose $A A$ is effective against both early and late phases of oxidative stress injury. Therefore, it can be more preferential in clinical practice. 
Previous reports indicated that AA had a dose-dependent protective effect against nephrotoxicity ${ }^{23}$. In conjunction with this data, we observed that PCO was decreased in Group III that received high-dose AA; while PCO level was not decreased in Groups I and II after the procedure. Our results support that administration of high-dose AA may offer more favorable results against nephrotoxicity associated with anesthesia.

Rats receiving glycerol injection under sevoflurane anesthesia developed severe $A R F$, and this consequence was reflected as increased serum levels of blood urea nitrogen and creatinine. Nephrotoxicity ensourced from glycerol was alleviated with the administration of $A A$, and this protective effect was more evident with increased doses. These preventive effects were detected regarding both functional and structural parameters.

In the current study, the glycerolinduced renal damage was accompanied with significant diminution of antioxidant enzymes, and these results are consistent with previous publications ${ }^{12}$. It has been demonstrated that free radicals may play an important role in the pathogenesis of myoglobinuric $\mathrm{ARF}^{24}$.

Our results indicated that $A A$ enhanced TAC and attenuated the injury linked with oxidative stress, reflected as levels of NGAL, PCO, urea, and creatinine. These beneficial effects were more obvious with increased doses of AA. Therefore, we suggest that use of $A A$ as a preventive and therapeutic agent against acute renal failure in patients who are planned for sevoflurane anesthesia may be promising.

Accumulation of cast and fibrin in tubuli and necrosis reflects the intraluminal accumulation of heme and severe renal morphologic injury ${ }^{1}$. It has been postulated that variability between experimental groups regarding renal hemodynamics may influence the histopathological results. Interestingly, necrosis was less obvious in the group receiving a higher dose of AA compared to that having a lower dose of $A A$.

The protective effect of $A A$ can be attributed to its free radical scavenger effects. It reacts directly with physiologically relevant reactive oxygen and nitrogen species and reactive nitrogen species. Ascorbic acid breaks free radical chain reactions, decreases consumption of endogen antioxidant enzymes, and prevents important biological macromolecules such as lipids, proteins, and DNA from oxidative damage. Superoxide anion has a great affinity for nitric oxide (NO), and reduced bioavailability of NO, a potent renal vasodilator, can lead to renal hypoperfusion and tissue injury in the setting of myoglobinuric ARF. In other words, AA may improve NO bioavailability and potential renoprotective capacity through the inhibition of renal vasoconstriction in myoglobinuric ARF. Ascorbic acid may generate and enhance other antioxidants, including $\alpha$-tocopherol, glutathione, and carnitine. Furthermore, AA inhibits the formation of ferryl myoglobin, a toxic molecule that triggers lipid peroxidation ${ }^{12}$. Owing to its strong antioxidant features that inhibit formation of ferrly myoglobin, AA may have an advantage over other antioxidants in treating myoglobinuric ARF ${ }^{12}$.

The protective mechanism of $A A$ against postoperative nephrotoxicity may not be restricted to oxidative stress. Elucidation of the pathophysiology may provide development of novel agents that improve the safety of anesthetic protocols. It is impossible to establish a direct causal relationship in experimental animal models. Sevoflurane anesthesia displays a cytoprotective effect on proximal tubuli by attenuation of cytotoxic effects of myoglobin. Selection of the appropriate anesthetic protocol is critical for avoidance or at least minimization of morbidity in the postoperative period. Ascorbic acid may 
yield additional protective effect together with sevoflurane, which offers cytoprotection with release of subtoxic doses of inorganic fluoride ${ }^{1}$. Our results may possess significant clinical relevances particularly for patients with renal problems who will undergo general anesthesia with sevoflurane. Use of AA in selected cases who are likely to have rhabdomyolysis may diminish the risk of ARF in the postoperative period.

This study possesses certain limitations such as experimental design, small sample size, the complex cascade of oxidative stress mechanisms as well as subjectivity of histopathological examination. Regarding statistical analysis, the validity of chi-square results is under debate due to the small number of subjects in some subgroups. On the other hand, main strengths of the present trial involve simultaneous assessment of biochemical and histopathologic variables. The larger sample size may provide more significant results for parameters under investigation.

\section{Conclusions}

The ascorbic acid may serve as a prophylactic agent against renal damage in patients receiving sevoflurane anesthesia. Higher doses were associated with more apparent protective effects. However, determination of efficacy and optimal doses, as well as confirmation of the significance of our findings, necessitates further experimental and clinical trials on larger series.

\section{References}

1. Lochhead KM, Kharasch ED, Zager RA. Anesthetic effects on the glycerol model of rhabdomyolysis-induced acute renal failure in rats. J Am Soc Nephrol. 1998;9:305-9. PMID: 9527408.

2. Novis BK, Roizen MF, Aronson S, Thisted RA. Association of preoperative risk factors with postoperative acute renal failure. Anesth
Analg. 1992;78:143-9. PMID: 8267150.

3. Kharasch ED, Hankins DC, Thummel KE. Human kidney methoxyflurane and sevoflurane metabolism: Intrarenal fluoride production as a possible mechanism of methoxyflurane nephrotoxicity. Anesthesiology. 1995;82:689-99. PMID: 7879937.

4. Hollenberg NK, McDonald FR, Cotran R, Galvanek EG, Wahol M, Vandam LD, Merrill JP. Irreversible acute oliguric renal failure: a complication of methoxyflurane anesthesia. N Engl J Med. 1972;286:877-9. doi: 10.1056/ NEJM197204202861606.

5. Zager RA, Iwata M. Inorganic fluoride: divergent effects on human proximal tubular cell viability. Am J Pathol. 1997;150:735-45. PMID: 9033286.

6. Zager RA. Rhanbdomyolysis and myohemoglobinuric acute renal failure. Kidney Int. 1996;49:314-26. PMID: 8821813.

7. Kong HY, Zhu SM, Wang LQ, He Y, Xie HY, Zheng SS. Sevoflurane protects against acute kidney injury in a small-size liver transplantation model. Am J Nephrol. 2010;32:347-55. doi: 10.1159/000319623.

8. Lee HT, Ota-Setlik A, Fu Y, Nasr SH, Emala CW. Differential protective effects of volatile anesthetics against renal ischemiareperfusion injury in vivo. Anesthesiology. 2004;101:1313-24. PMID: 15564938.

9. Gentz BA, Malan TP Jr. Renal toxicity with sevoflurane: a storm in a teacup? Drugs. 2001;61:2155-62. PMID: 11772127.

10.Ansar S, lqbal M. Role of ascorbic acid in counteracting ferric nitrilotriacetateinduced nephrotoxicity in rats. Pharmaceutical Biol. 2013;51:1559-63. doi: 10.3109/13880209.2013.802811.

11.Yousef JM, Chen G, Hill PA, Nation RL, $\mathrm{Li} \mathrm{J}$. Ascorbic acid protects against the nephrotoxicity and apoptosis caused by colistin and affects its pharmacokinetics. J Antimicrob Chemother. 2012;67:452-9. PMID: 22127588.

12.Ustundag S, Yalcin O, Sen S, Cukur Z, Ciftci $S$, Demirkan B. Experimental myoglobinuric acute renal failure: the effect of vitamin C. Renal Failure. 2008;30:727-35. doi: $10.1080 / 08860220802212965$.

13.Ding H, He Y, Li K, Yang J, Li X, Lu R, Gao W. Urinary neutrophil gelatinase-associated lipocalin (NGAL) is an early biomarker 
for renal tubulointerstitial injury in IgA nephropathy. Clin Immunol. 2007;123:22734. doi: 10.1016/j.clim.2007.01.010.

14.Dridi I, Ben-Cherif W, Chahdoura $\mathrm{H}$, Haouas $Z$, Ben-Attia M, Aouam K, Reinberg A, Boughattas NA. Dosing-time dependent oxidative effects of an immunosuppressive drug "MycophenolateMofetil" on ratkidneys. Biomed Pharmacother. 2017;87:509-18. doi: 10.1016/j.biopha.2016.12.087.

15.Gulmen S, Kiris I, Narin C, Ceylan BG, Mermi $B$, Sutcu R, Meteoglu I. Tezosentan reduces the renal injury induced by abdominal aortic ischemia reperfusion in rats. J Surg Res. 2009;157:e7-13. doi: 10.1016/j. jss.2008.08.011.

16.Talke $H$, Schubert GE. Enzymatic urea determination in the blood and serum in the Warburg optical test. Klin Wochenschr. 1965;43:174-5. PMID: 14258517.

17.Fabiny DL, Ertinghausen G. Automated reaction-rate method for determination of serum creatinine with the Centrifichem. Clin Chem. 1971;17:696-700. PMID: 5562281.

18.Atashpour S, Jahromi HK, Jahromi ZK, Zarei S. Antioxidant effects of aqueous extract of Salep on Paraquat-induced rat liver injury. World J Hepatol. 2017;9:209-16. doi: 10.4254/wjh.v9.i4.209.

19.Reznick AZ, Packer L. Oxidative damage to proteins: spectrophotometric method for carbonyl assay. Methods Enzymol. 1994;233:357-63. PMID: 8015470.

20.Ding H, He Y, Li K, Yang J, Li X, Lu R, Gao W. Urinary neutrophil gelatinase-associated lipocalin (NGAL) is an early biomarker for renal tubulointerstitial injury in IgA nephropathy. Clin Immunol. 2007;123:22734. doi: 10.1128/CVI.00335-07.

21.Patel RK, Trivedi AH, Roy SK, Bhatavdekar JM, Shah PM, Patel DD. Influence of alphatocopherol and adcorbic acid on panmasala induced genomic damage. An in vitro experiment. J Exp Clin Cancer Res. 1999;17:419-24. PMID: 10089061.

22.Taniguchi M, Arai N, Kohno K, Fukuda S. Anti-oxidative and anti-aging activities of 2-O-a-glucopyranosyl-L-ascorbic acid on human dermal fibroblasts. Eur J Pharmacol. 2012;15:674:126-31. PMID: 22119379.

23.Antunes LM, Darin JD, Bianchi MD. Protective effects of vitamin $c$ against cisplatin-induced nephrotoxicity and lipid peroxidation in adult rats: a dose-dependent study. Pharmacol Res. 2000;41:405-11. doi: 10.1006/phrs.1999.0600.

24.Shah SV, Walker PD. Evidence suggesting a role for hydroxyl radical in glycerolinduced acute renal failure. Am J Physiol. 1988;255:F438-43. PMID: 2843051.

\section{Correspondence:}

Omur Ozturk

Department of Anesthesiology and Reanimation Canakkale Onsekiz Mart University, Faculty of Medicine

Canakkale Turkey

Phone: +905325135709

dromur52@hotmail.com

Received: June 16, 2017

Review: Aug 14, 2017

Accepted: Sept 18, 2017
Conflict of interest: none

Financial source: none
${ }^{1}$ Research performed at Laboratory Animal Research Center, Kafkas University, Kars, Turkey. 